\title{
Incremental Accessibility Benefits and Choice of Subscriptions for High-Occupancy Toll Lanes
}

\author{
Andrew Owen, Michael Janson, and David M. Levinson
}

\begin{abstract}
This paper presents the results of an investigation into the factors contributing to toll lane subscription choice by using data from the MnPASS high-occupancy toll lane system operated by the Minnesota Department of Transportation. The paper estimates a binomial logit model that predicts, on the basis of aggregate characteristics of the surrounding area, the likelihood of a household having a subscription to MnPASS systems. Variables in this model include demographic factors as well as an estimate of the incremental accessibility benefit provided by the MnPASS system. This benefit is estimated with the use of detailed accessibility calculations and represents the degree to which a location's accessibility to jobs is improved if HOT lanes are available. The model achieves a $\rho^{2}$ value of .634, and analysis of the results suggests that incremental accessibility benefits play a statistically and practically significant role in determining how likely households are to hold a toll lane subscription.
\end{abstract}

The majority of research related to high-occupancy toll (HOT) lanes and other types of toll lanes takes place at the route choice level, investigating the factors that cause travelers to select a toll lane during a single trip or as part of a typical commute route. However, the electronic toll collection (ETC) systems commonly used in urban toll lane systems add another layer to travelers' decision-making process that is typically not considered explicitly. ETC systems typically require travelers to carry transponders, cards, or similar devices if they plan to use electronically tolled facilities. This equipment is typically available at low or no cost, but there are no known instances of toll lane systems where transponders are provided preemptively; enrollment in toll lane systems requires some action or expense by potential users. Even before a traveler is presented with the option of entering a toll lane, he or she must decide to become an eligible HOT lane user.

This analysis investigated the factors contributing to toll lane subscription choice by using data from the MnPASS HOT lane system operated by the Minnesota Department of Transportation (DOT). The paper estimates a binomial choice model that predicts, on the basis of aggregate characteristics of the surrounding area, the likelihood of a household having a subscription to the MnPASS system. Variables in this model include demographic factors as well as an estimate of the incremental accessibility benefit provided by the MnPASS system. This benefit is estimated at the level of the transportation analysis zone (TAZ) level by using detailed accessibility calculations. The

Department of Civil Engineering, University of Minnesota, 500 Pillsbury Drive SE, Minneapolis, MN 55455. Corresponding author: A. Owen, aowen@umn.edu.

Transportation Research Record: Journal of the Transportation Research Board, No. 2412, Transportation Research Board of the National Academies, Washington, D.C., 2014, pp. 93-99.

DOI: $10.3141 / 2412-11$ authors hypothesize that, in areas where access to the MnPASS system would provide greater accessibility to jobs, households are more likely to have MnPASS subscriptions.

The next section provides a background discussion of the MnPASS system and toll lanes in general, as well as the application of accessibility evaluation and transportation choice modeling in this context. Subsequent sections describe the data and methods employed in this analysis, followed by a presentation and discussion of the model results and performance.

\section{BACKGROUND}

\section{MnPASS System}

Minnesota DOT began operating eastbound and westbound HOT lanes on I-394 west of downtown Minneapolis in May 2005. In 2009, the system was expanded to include northbound and southbound HOT lanes on I-35W south of downtown Minneapolis. Figure 1 illustrates the location of MnPASS lanes within the Twin Cities area. During peak periods, access to these lanes is restricted to vehicles with two or more occupants and those paying a toll for access. Outside peak periods, the lanes are open to general traffic. The toll varies from $\$ 0.25$ to $\$ 8.00$, and is dynamically adjusted on the basis of the current utilization of MnPASS lanes. Tolls are collected electronically; vehicles must carry a transponder that is provided by Minnesota DOT after participants enroll through a website, by mail, or in person. Transponders are leased for $\$ 1.50 /$ month (1-3).

\section{Toll Lanes and ETC}

Toll lanes using transponder-based ETC are a relatively young transportation technology, and data collection methods have become established only over the past decade. Li et al. (4) and Li et al. (5) provided a template for analyzing HOT lane usage and behavior by using two important sources of data: toll collection records, which capture every interaction that subscribers have with the HOT lane system, and GPSbased surveys, which capture detailed trip logs, regardless of whether a HOT lane is used. For full understanding of traveler behavior in a HOT lane system, both must be used, because toll collection records alone cannot reveal traveler behavior when a HOT lane is not used. (However, some ETC systems, such as Georgia's PeachPass, employ transponder readers in nontolled lanes to provide this information.)

Burris and Stockton highlighted the importance of understanding the economics and behavior associated with HOT lanes (6). They chronicled the results of several variations of HOV and HOT policies in Houston, Texas, where HOT lane pricing was implemented as a 
tool for more effectively guiding an appropriate level of demand into the HOT lane while preserving travel time reliability. This importance is compounded because traveler behavior in HOT lane systems is often counterintuitive, as illustrated by Liu et al., in which higher HOT lane prices were found to increase demand (7). Janson and Levinson found similar results through an investigation of user behavior on the MnPASS HOT lanes (8), which included experimental price modifications in the field. Goodall and Smith applied transportation choice theory in the context of HOT lanes (2). Their findings suggest that travel time reliability, rather than expected travel time savings, drives both the choice to enroll in HOT lane programs and the choice to use a HOT lane on a per-trip basis. Small et al. (9) and Brownstone and Small (10) also investigated travelers' valuation of travel time and reliability.

These and many other studies focused on the choices made by travelers when they decide whether to use a toll lane during an individual trip. But electronic toll collection systems add another layer of choice to the decisions facing potential toll lane users: whether to enroll in the system. Enrolling in a HOT lane system is in most cases low cost or free financially, but it always requires some expense from users in time spent processing enrollment forms and managing accounts. In addition, enrollment involves some risk: electronic toll systems typically charge users for lost or damaged transponders (11). The importance of the subscription choice was recognized by Minnesota DOT in its consideration of how (and whether) to market the I-294 MnPASS lanes when they began operation in 2005. It recognized that, because a subscription is required to use the systems, the number of subscribers effectively caps the maximum demand that the system could experience $(2,12)$.

Parkany directly investigated subscription choice in the context of tolled express lanes in Orange County, California, applying a logitbased choice model (13). The findings established that having a toll lane subscription is more determinative of toll lane use than demographic or trip characteristic factors. A later study by Parkany examined more closely the role that enrollment costs play in determining how much, when, and by whom toll lanes are used (11). Similarly, Levinson and Chang incorporated the cost (both monetary and temporal) of enrollment in a model designed to maximize the social welfare of an ETC system and found these costs to be a key factor in toll lane usage and outcomes (14).

Because of the costs (however small) associated with subscribing to a toll lane program, expectations are reasonable that people who would not benefit from the ability to use HOT lanes would not enroll in a HOT lane toll collection program. Conversely, intuitively, a person who would receive an extremely large benefit from enrollmentperhaps a commute duration that is much shorter or much more reliable-would be extremely likely to enroll.

\section{Accessibility}

Accessibility combines the simpler concept of mobility with an understanding that travel is driven by a desire to reach destinations. Distinguishing between "individual accessibility" and "locational accessibility" is important: the former seeks to characterize the ease with which travelers might reach their destinations, subject to constraints of ability, budget, and other barriers; the latter examines accessibility as a spatial phenomenon by considering the costs and benefits of the potential trips offered by transportation systems between origins and destinations of interest.

Horner explored this distinction in the literature and noted that individual accessibility measures are generally poor at "producing... generalized assessments of intraurban structure," while location accessibility measures are more useful for "understanding relationships between transportation and land use" (15). Locational accessibility can be a particularly useful tool for transportation planners because it provides a way to evaluate the properties of transportation systems at a level that is sufficiently aggregate to avoid the vagaries of individual users' preferences and constraints but sufficiently detailed to provide guidance for planning at the city and regional levels.

Many implementations of locational accessibility measurement are possible. El-Geneidy and Levinson provided a practical overview of historical and contemporary approaches (16). Perhaps the simplest approach to evaluating locational accessibility was discussed by Ingram (17) and by Morris et al. (18): "cumulative opportunity" measures of accessibility employ a binary weighting function. Accessibility is calculated for specific time thresholds, and the result is a simple count of destinations that are reachable within each threshold. The current analysis employs a cumulative-opportunities measure of accessibility that follows the method described in Minnesota DOT's Access to Destinations research project (19).

\section{DATA}

\section{Road Network}

The Metropolitan Council, the metropolitan planning organization for the Twin Cities region, maintains a model of the regional road network. It provides a network topography for freeway, arterial, and collector roadways in the region that is designed to model travel by using TAZs as origins and destinations. The most recent version of this network was updated in 2009 , and it provides an adequately accurate representation of the state of the regional road network as of 2010, with model links conflated to match actual geometry. It does not model local roads but instead provides dummy links that connect the centroid of each TAZ to adjacent arterial links. These links are coded so that they may be used only for direct access to or from a TAZ centroid; they may not be used for travel between zones.

\section{Speeds}

The Twin Cities' regional freeway network is extremely well instrumented, and data recorded by loop detectors throughout the system are archived by the Minnesota Traffic Observatory, operated and hosted by the University of Minnesota. Archived loop detector data for every weekday in 2010 provided the basis for average freeway link speeds.

Freeway speeds are derived from direct traffic observations made by embedded loop detectors, which record the observed traffic volume and detector occupancy at 30-s intervals. Minnesota DOT provides an estimated average effective vehicle length for each detector, which allows the calculation of speed from volume and occupancy. These 30-s speed measurements are aggregated by averaging 5-min time slots. Finally, the 5-min speed measurements taken between 7 and 9 a.m. on weekdays during 2010 are averaged for each detector to produce a representation of average speeds during morning peak periods. The resulting detector speeds are assigned to links in the model on the basis of location.

In contrast to freeways, local arterials and collectors are only sparsely instrumented. Average arterial and collector link speeds are estimated from speed measurements made during a regional GPS-based travel survey in 2008 (20). These data represent an extremely accurate measurement of traffic speeds at specific locations 
and specific times. Speeds for unobserved links are estimated from the samples collected on links with a similar functional classification.

\section{HOT Lane Subscriptions}

Information describing the home location of MnPASS subscribers is collected as billing addresses associated with each MnPASS account. MnPASS users initially provide this information when they create an account, and the information is, of necessity, updated when credit car billing addresses change. Multiple vehicles may be associated with a single account, and the billing address need not be a home addressand drivers of the associated vehicles need not be related to the account holder. For example, a single MnPASS subscriber might use a business address as the billing address, or a business might create an MnPASS account by using a single billing address to request multiple MnPASS transponders for a fleet of vehicles (3). These facts have important implications for the use and interpretation of information on MnPASS subscriber locations. In particular, in some TAZs, the ratio of MnPASS accounts to households is questionably high and is occasionally greater than 1 . To address this situation, the models presented in this analysis exclude TAZs in which the number of MnPASS accounts is greater than $50 \%$ of the number of households. Of 2,370 TAZs with one household, this situation occurred in only $20(0.8 \%)$, where the ratio of MnPASS accounts to households was 8.56 standard deviations or more above the mean. One may reasonably suspect that among these outliers a significant number of billing addresses for MnPASS accounts are not home addresses. Block groups with no households are also excluded. This method is not ideal: it excludes some household accounts in TAZs with many business accounts, and it includes some business accounts in TAZs in which the account- household ratio is low. However, it makes the best use of the available account location data.

To support this research project, Minnesota DOT provided a data set derived from the billing addresses for MnPASS subscribers as of mid-2012. This data set provides a count of billing addresses for MnPASS accounts that fall within each TAZ. Figure 1 illustrates these data as the ratio of billing addresses for MnPASS accounts to households in each TAZ.

\section{Regional Demographics}

Demographic information describing the residents of the Minneapolis-Saint Paul metropolitan area is used to associate aggregate demographic characteristics with geographic areas for use in modeling MnPASS subscription choice. This analysis uses estimates for 2007 to 2011 from the U.S. Census Bureau's American Community Survey (ACS).

\section{Employment}

Data describing the distribution of employment in the region are drawn from the U.S. Census Bureau's Longitudinal EmployerHousehold Dynamics (LEHD) program. The workplace area characteristic data set for 2011 provides estimates at the census block level for employee home and work locations.

In general, LEHD is a quite useful data source for accessibility evaluation because it is updated yearly and is drawn from actual payroll records collection at the state level — in this case, by the Minnesota Department of Employment and Economic Development. However,

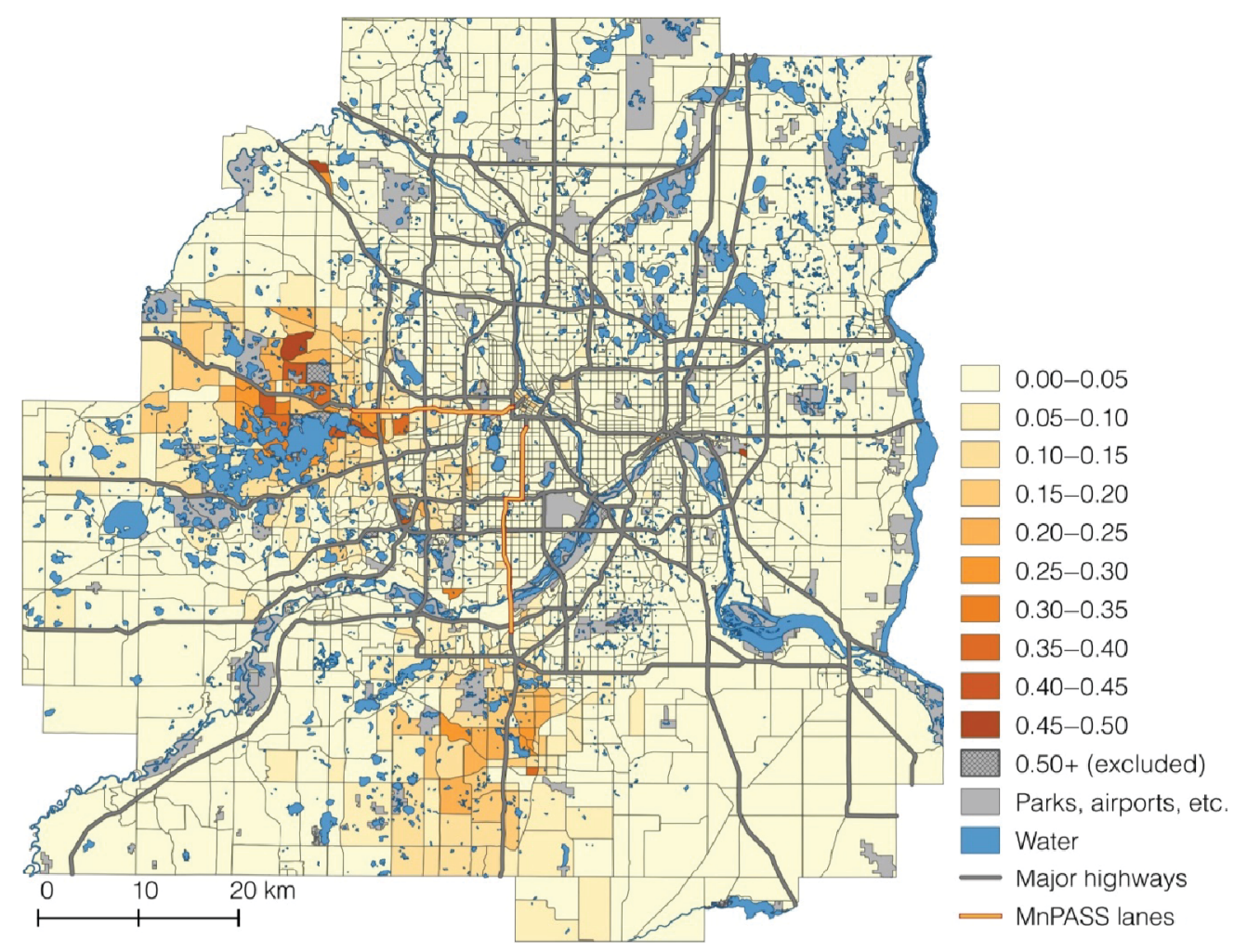

FIGURE 1 MnPASS accounts per household, by TAZ. (Source: Minnesota DOT, American Community Survey 2007 to 2011 estimates.J 
LEHD data are synthetic: while it is based on actual payroll records, the published results are created by an algorithm designed to produce data that are statistically similar to the underlying data and that converge to the same distribution when aggregated. An analysis by Spear of LEHD data in transportation analysis found LEHD to be a useful source of both home and work location data but identified shortcomings related to job locations of federal workers (21). Tilahun and Levinson demonstrated the use of LEHD data in contemporary transportation research (22).

\section{METHODOLOGY}

\section{Calculating Accessibility}

Transportation choice theory suggests that the decision to subscribe to MnPASS is based in part on the benefits that the MnPASS system provides to its subscribers. Here, this benefit is represented by the increase that residents experience in accessibility to jobs when they gain access to MnPASS lanes.

Accessibility calculation begins with the construction of a travel time matrix that indicates the shortest-path travel time between all pairs of TAZs. Travel times by auto are calculated by using a shortestpath search on a graph defined by the Metropolitan Council's 2009 regional road network model and the speed data sources described above. All speeds are measurements or estimates of average link speeds during the period between 7 and 9 a.m.

Auto travel times do not include an estimation of access times at the origin (e.g., walking from home to a car) or at the destination (e.g., walking from a parking space to work). While the former is likely negligible given the typical residential urban forms in the study area, the latter has the potential to be significant. Unfortunately, the spatial resolution of the road and highway network model available for this analysis is not sufficiently detailed to allow accurate estimation of destination access times.

Through the travel time matrices described earlier, accessibility of cumulative opportunities to jobs is calculated for each origin by using a time threshold of $30 \mathrm{~min}$. For each origin, this process identifies all destinations reachable within $30 \mathrm{~min}$ and sums the number of jobs at those destinations. The 30-min threshold was chosen on the basis of ACS's 2007 to 2011 estimate that $80 \%$ of workers in the Twin Cities area have commutes of 30 to 34 min or less.

Two sets of accessibility values are calculated. The first represents the case in which a traveler is not an MnPASS subscriber and therefore does not have access to the MnPASS lanes. In this scenario, the speeds for all links are derived from the measurements and estimates described earlier. Figure 2 illustrates the patterns of accessibility in this scenario (in the figure, higher values indicate that a greater number of jobs can be reached by auto within 30 min during the morning peak period and assume no use of MnPASS lanes). The second scenario represents the case in which a traveler is an MnPASS subscriber and therefore has the option of using MnPASS lanes. In this scenario, the speeds for freeway segments where MnPASS lanes are available are set to $65 \mathrm{mph}$, a typical speed observed by detectors monitoring those lanes.

These two accessibility data sets are combined to create a representation of the incremental accessibility benefits provided by the MnPASS system. The benefit is represented by the ratio of nonMnPASS accessibility to MnPASS accessibility. In locations where the MnPASS lanes provide no benefit, these values are equal and their ratio is 1.0. As the benefit from MnPASS lane access increases, this ratio decreases. In the TAZs where the MnPASS lanes provide the

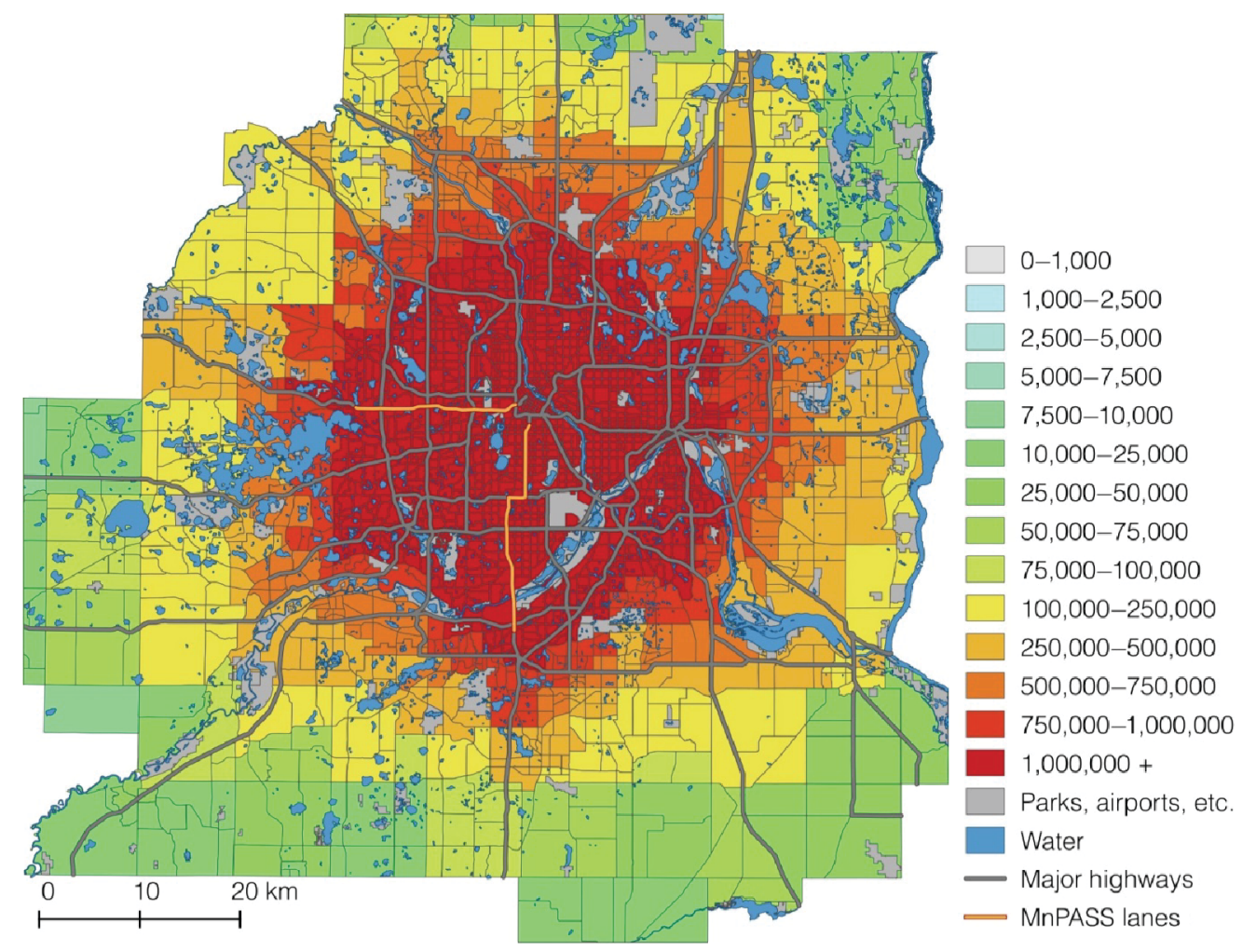

FIGURE 2 Thirty-minute accessibility to jobs by auto. 


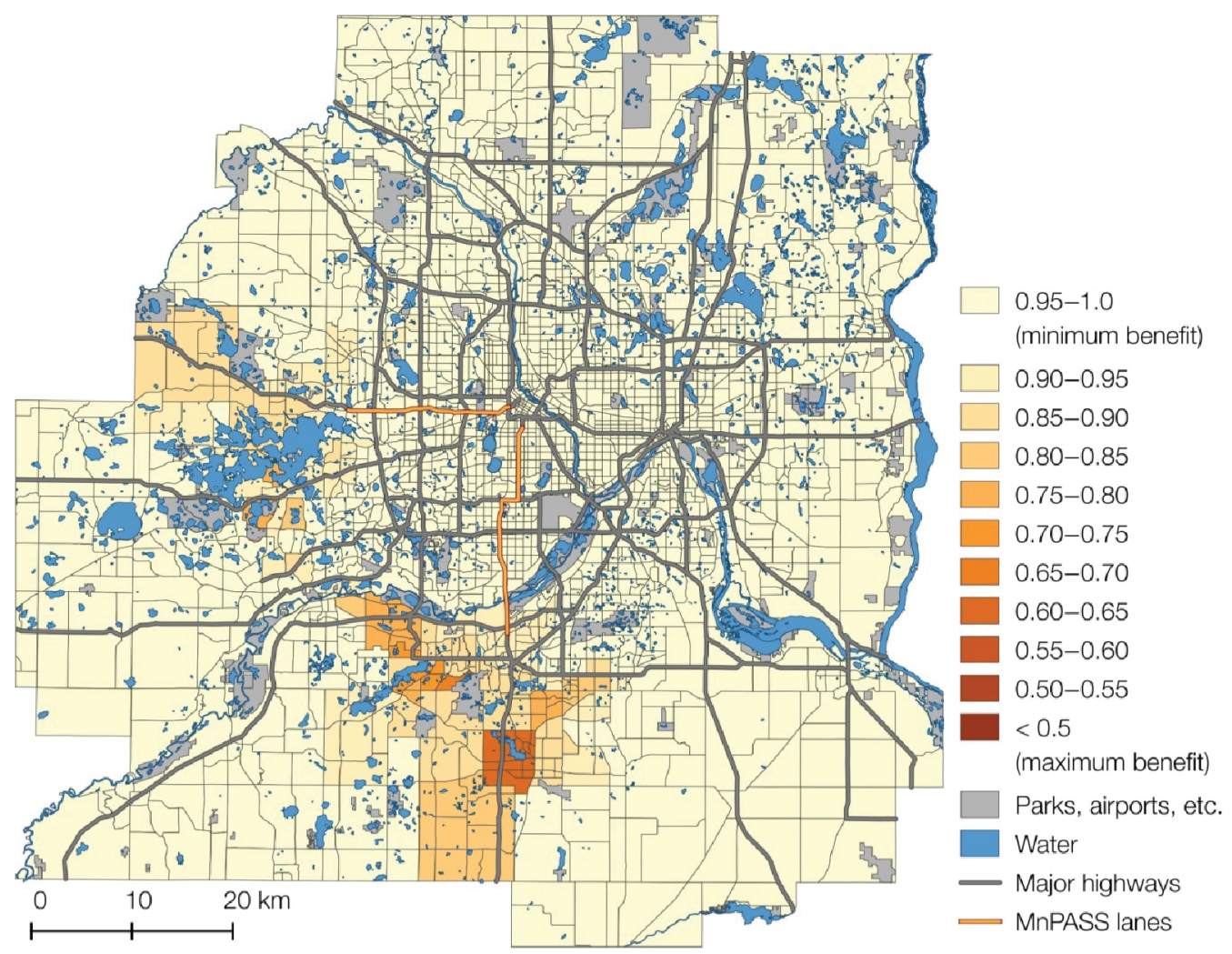

FIGURE 3 Accessibility benefit produced by MnPASS system.

greatest benefit, this ratio is .62 , indicating that non-MnPASS accessibility is $62 \%$ of MnPASS accessibility. Other expressions of the incremental accessibility benefit, including the difference and the reciprocal ratio, were evaluated; this ratio provides the best model performance. Figure 3 illustrates the incremental accessibility benefit provided by the MnPASS system throughout the Twin Cities area.

\section{Model Formulation}

The subscription choice model predicts the likelihood that a given household has access to an MnPASS account on the basis of aggregate local demographics and on the local incremental accessibility benefit provided by the MnPASS system. The models are constructed on the basis of a binomial choice: a household can either have or not have an MnPASS pass account. This choice is modeled by using the binomial logit form, where the likelihood of an individual making each choice depends on the utility indicated by a specified set of variables.

The subscription choice model includes independent variables that fall into two classes: those that describe properties of a household's location (specifically, the TAZ in which it falls), and those that describe demographic properties of the household's TAZ. These variables are summarized in Table 1 and described in the following paragraphs.

Locational variables include the following:

- Distance to MnPASS entry point $(D)$ reflects the distance (in kilometers) from a household's TAZ to the nearest MnPASS entry point. An MnPASS entry point is defined as a freeway entrance ramp that could be used to access an MnPASS lane for a trip toward downtown Minneapolis. Distances are measured along the road network.
- Baseline accessibility $\left(A_{M}\right)$ indicates the number of jobs, in hundred thousands, reachable within 30 min using only general-purpose lanes from a household's TAZ during the morning peak period.

- Accessibility benefit ratio $(R)$ reflects the increase in accessibility to jobs that the MnPASS lanes provide to the household's TAZ, expressed as the ratio of non-MnPASS accessibility:

$R=\frac{A_{M}}{\text { accessibility using MnPASS lanes }}$

Demographic variables include these:

- Average household size $(S)$ reflects the size (number of persons) of a household in a TAZ,

TABLE 1 Independent Variables in Subscription Choice Models

\begin{tabular}{llcr}
\hline Variable & Description & Mean $^{a}$ & \multicolumn{1}{c}{$\begin{array}{l}\text { Standard } \\
\text { Deviation }^{a}\end{array}$} \\
\hline$D$ & $\begin{array}{l}\text { Distance to MnPASS entry } \\
\text { point (km) }\end{array}$ & 16.18 & 11.93 \\
$\quad \begin{array}{c}\text { Baseline accessibility } \\
\text { (no MnPASS, 100,000 jobs) }\end{array}$ & 869.56 & 491.02 \\
& Accessibility benefit ratio & 0.98 & 0.05 \\
$R$ & Average household size & 2.58 & 0.50 \\
$S$ & Average household income & $\$ 93,686$ & $\$ 41,652$ \\
$I$ & Average number of vehicles & 1.91 & 0.47 \\
$V$ & per household & & \\
\hline
\end{tabular}

${ }^{a} n=2,350$ TAZs. 
- Average household income $(I)$ reflects the total household income of the households in a TAZ, and

- Average number of vehicles per household $(V)$ reflects the number of vehicles available per household in a TAZ.

Models are constructed by using various combinations of these variables to investigate their relative strengths as descriptors of the likelihood of subscription to MnPASS. Model 1 includes only demographic variables; Models 2 and 3 include MnPASS entry point proximity and incremental accessibility benefits individually, and Model 4 incorporates all variables. Furthermore, each model includes the estimation of an alternative-specific constant to capture the variation in transponder ownership that is not explained by the earlier-mentioned parameters.

\section{Model Evaluation}

To evaluate the several models that will be presented, one must be able to measure two things: first, evaluation of the fit between each model and the data and, second, evaluation of the performance of each model relative to others.

Model fit is evaluated by using the pseudo- $R^{2}$ for logistic regression labeled as $\rho^{2}$ by McFadden and calculated from the likelihood measures of the specified model and the null model $(23,24)$. In an evaluation of pseudo- $R^{2}$ measures in the context of logistic regression, Menard identified several desirable qualities of $\rho^{2}(25)$. Most importantly, $\rho^{2}$ was found to have an "intuitively reasonable interpretation as a proportional reduction in error measure" (25).

Relative model performance is evaluated by using the Akaike information criterion (AIC), which estimates the amount of information lost by using the model instead of the data and penalizes models for including more parameters. The penalty property is important given that this analysis depends on comparisons between models with different numbers of parameters (26).

\section{RESULTS AND DISCUSSION}

Table 2 summarizes the results of the subscription choice models. Model 4, which includes the full set of tested variables, provides the best fit for the data $\left(\rho^{2}=.634\right)$ and the best performance relative to all other models (AIC $=22,037)$. All the variables, both locational and demographic, have a high statistical significance in all models.

The performance of Model 1 is quite low compared with the others. This is unsurprising given the variables included: it uses only demographic variables, omitting any description of household location. It is intuitive that the benefit a household receives from the ability to use MnPASS lanes has some relationship to its location relative to the lanes, and this model does not account for that effect. However, the demographic variables have a high statistical significance in all models. This aspect suggests that, given two households in the same location, demographic factors still play an important role in MnPASS subscription likelihood.

Higher average household income $(I)$ and greater number of vehicles per household $(V)$ are associated with increased likelihood of MnPASS enrollment. This observation is consistent with existing findings that the perceived value of travel time savings and increased travel time reliability increased with individuals' income. The association with income may also be related to the effects identified by Parkany (11) and Levinson and Chang (14), who found that the enrollment costs and requirements of ETC systems make them less attractive or less available to low-income individuals regardless of the benefits that could be realized. Alternatively, the work of Patterson proposes that the income relationship may exist simply because HOT lanes serve high-income areas more frequently or more thoroughly than low-income areas and thereby generates a spatial correlation that does not necessarily reflect user preferences (27).

The effect of vehicle availability has two apparent interpretations. First, higher vehicle ownership suggests a higher value of travel by auto and therefore a highervalue of the decreased auto travel times provided by the MnPASS systems. Second, each additional vehicle available to a household effectively represents an additional opportunity or motivation to enroll in the MnPASS system.

Models 2, 3, and 4 illustrate the relative importance of and the relationship between accessibility to a transportation system and accessibility via a transportation system. The distance to the nearest MnPASS entry point $(D)$ is a representation of the ease of reaching the MnPASS system itself, while the accessibility measures $\left(A_{M}\right.$ and $R$ ) indicate the ease of reaching destinations (in this case, jobs) by using the MnPASS system. The model results suggest that both are important factors in determining the likelihood that a household will hold an MnPASS subscription. In Models 2 and 4, the negative coefficient of $D$ indicates that the likelihood of transponder ownership decreases for TAZs that are farther from an MnPASS entry point. The inclusion of either MnPASS proximity or accessibility benefit (Models 2 and 3) provides a roughly equivalently significant improvement in model performance over Model 1, and including both (Model 4) provides another significant improvement.

The coefficient associated with the incremental accessibility benefit $(R)$ must be interpreted carefully. An $R$ value of 1.0 indicates no

TABLE 2 Results of Subscription Choice Models

\begin{tabular}{|c|c|c|c|c|c|c|c|c|}
\hline \multirow[b]{2}{*}{ Variable } & \multicolumn{2}{|c|}{ Model 1} & \multicolumn{2}{|c|}{ Model 2} & \multicolumn{2}{|c|}{ Model 3} & \multicolumn{2}{|c|}{ Model 4} \\
\hline & Value & $z$ & Value & $z$ & Value & $z$ & Value & $z$ \\
\hline$D$ & - & - & -0.112 & $-98.69 *$ & - & - & -0.161 & $-90.00 *$ \\
\hline$A_{M}$ & - & - & - & - & 0.069 & $29.64 *$ & -0.182 & $-56.94 *$ \\
\hline$R$ & - & - & - & - & -0.113 & $-123.95^{*}$ & -0.355 & $-29.12 *$ \\
\hline$S$ & -0.559 & $-28.00 *$ & -0.269 & $-13.51 *$ & -0.717 & $-35.28 *$ & -0.484 & $-23.32 *$ \\
\hline$I$ & 0.013 & $108.19 *$ & 0.008 & $58.12 *$ & 0.012 & $87.21 *$ & 0.010 & $74.75^{*}$ \\
\hline V & 0.680 & $26.25^{*}$ & 1.594 & $56.91^{*}$ & 0.830 & $26.82 *$ & 0.679 & $21.95^{*}$ \\
\hline ASC & -5.027 & $-133.77 *$ & -5.819 & $-146.28 *$ & 5.464 & $55.25^{*}$ & 1.755 & $15.62 *$ \\
\hline
\end{tabular}

NotE: - indicates variable not included. $\rho^{2}$, AIC, and $n$, respectively: Model $1=.246,45,392,2,350$; Model $2=.474,31,581$, 2,650; Model $3=.461,32,461,2,350 ;$ Model $4=.634 ; 22,037,2,350$.

* Significant at $0.1 \%$ level or better. 
benefit, and lower values indicate greater benefit. The coefficient -.355 indicates that, as a TAZ's ratio of non-MnPASS accessibility to MnPASS accessibility increases by .01, a household in that TAZ is only $.701\left(=e^{-0.355}\right)$ times as likely to have an MnPASS subscription. Conversely, a decrease in this ratio, indicating greater accessibility benefit from the MnPASS system, signifies an increased subscription likelihood - supporting this investigation's hypothesis.

The negative coefficient associated with baseline non-MnPASS accessibility $\left(A_{M}\right)$ is counterintuitive but reasonable upon consideration. As illustrated in Figure 2, non-MnPASS accessibility in the region is strongly central: the highest values are found near downtown Minneapolis, and accessibility declines fairly consistently with distance from that point. The existing MnPASS facilities are almost perfectly radial, so locations near their suburban ends have lower non-MnPASS accessibility than locations near their central ends.

\section{CONCLUSION}

These results suggest that the evaluation of incremental accessibility benefits can be a useful tool in modeling participation in toll lane programs. Inclusion of this accessibility measure provides a significant reduction in model error compared with models that use only demographics and those that also include locational variables. By incorporating incremental accessibility benefits, a relatively straightforward model is able to describe a significant portion of the variation in aggregate toll lane subscription choice rates.

It is likely that equal or better model performance could be achieved by modeling subscription choice at the individual level, using detailed knowledge of commute destinations, routes, and times. However, this information is typically costly to acquire and process and often raises issues of privacy. Aggregate-choice approaches, informed by detailed accessibility calculations, can facilitate useful modeling and forecasting in the absence of such data.

This direct model of HOT lane subscription choice can be applied in the design and planning of new and expanded HOT lane facilities. By using current conditions as a baseline, one may calculate the incremental accessibility benefits provided by new HOT lane infrastructure on the basis of expected HOT lane speed. This type of analysis can help reveal not only the benefits a HOT lane provides but also the areas that experience those benefits and the likelihood of residents participating in the HOT lane program. Earlier research suggests that having a subscription, transponder, or similar HOT lane access device can itself be a strong predictor of HOT lane use, so the results can be further applied to inform projections of HOT lane use.

\section{REFERENCES}

1. Turnbull, K. F., K. Buckeye, and N. Thompson. I-35W South MnPASS High-Occupancy Toll Lanes. Presented at 92nd Annual Meeting of the Transportation Research Board, Washington, D.C., 2013.

2. Goodall, N., and B. L. Smith. What Drives Decisions of Single-Occupant Travelers in High-Occupancy Vehicle Lanes?: Investigation Using Archived Traffic and Tolling Data from MnPASS Express Lanes. In Transportation Research Record: Journal of the Transportation Research Board, No. 2178, Transportation Research Board of the National Academies, Washington, D.C., 2010, pp. 156-161.

3. MnPASS. Minnesota Department of Transportation, Saint Paul, 2013. http://www.mnpass.org. Accessed Aug. 1, 2013.

4. Li, H., R. Guensler, J. Ogle, and J. Wang. Using Global Positional System Data to Understand Day-to-Day Dynamics of Monitoring Commute
Behavior. In Transportation Research Record: Journal of the Transportation Research Board, No. 1895, Transportation Research Board of the National Academies, Washington, D.C., 2004, pp. 78-84.

5. Li, H., R. L. Guensler, and J. H. Ogle. Analysis of Morning Commute Route Choice Patterns Using Global Positioning System-Based Vehicle Activity Data. In Transportation Research Record: Journal of the Transportation Research Board, No. 1926, Transportation Research Board of the National Academies, Washington, D.C., 2005, pp. 162-170.

6. Burris, M. W., and B. R. Stockton. HOT Lanes in Houston: Six Years of Experience. Journal of Public Transportation, Vol. 7, 2004, pp. 1-22.

7. Liu, X., G. Zhang, Y. Lao, and Y. Wang. Quantifying the Attractiveness of High-Occupancy Toll Lanes with Traffic Sensor Data Under Various Traffic Conditions. In Transportation Research Record: Journal of the Transportation Research Board, No. 2229, Transportation Research Board of the National Academies, Washington, D.C., 2011, pp. 102-109.

8. Janson, M., and D. Levinson. HOT or Not: Driver Elasticity to Price on the MnPASS HOT Lanes. Research in Transport Economics, forthcoming.

9. Small, K. A., C. Winston, and J. Yan. Uncovering the Distribution of Motorists' Preferences for Travel Time and Reliability. Econometrica, Vol. 73, No. 4, 2005, pp. 1367-1382.

10. Brownstone, D., and K. A. Small. Valuing Time and Reliability: Assessing the Evidence from Road Price Demonstrations. Transportation Research Part A: Policy and Practice, Vol. 39, No. 4, 2005, pp. 279-293.

11. Parkany, E. Environmental Justice Issues Related to Transponder Ownership and Road Pricing. In Transportation Research Record: Journal of the Transportation Research Board, No. 1932, Transportation Research Board of the National Academies, Washington, D.C., 2005, pp. 97-108.

12. Munnich, L. W., Jr., and K. R. Buckeye. I-394 MnPASS High-Occupancy Toll Lanes: Planning and Operational Issues and Outcomes (Lessons Learned in Year 1). In Transportation Research Record: Journal of the Transportation Research Board, No. 1996, Transportation Research Board of the National Academies, Washington, D.C., 2007, pp. 49-57.

13. Parkany, E. Modeling the Hurdle of Obtaining a Transponder on the 91 Express Lanes. Presented at 10th Meeting of Intelligent Transportation Society of America, Washington, D.C., 2000.

14. Levinson, D., and E. Chang. A Model for Optimizing Electronic Toll Collection Systems. Transportation Research Part A: Policy and Practice, Vol. 37, No. 4, 2003, pp. 293-314.

15. Horner, M. W. Spatial Dimensions of Urban Commuting: A Review of Major Issues and Their Implications for Future Geographic Research. Professional Geographer, Vol. 56, No. 2, 2004, pp. 160-173.

16. El-Geneidy, A. M., and D. M. Levinson. Access to Destinations: Development of Accessibility Measures. MN/RC 2006-16. Minnesota Department of Transportation, Saint Paul, 2006.

17. Ingram, D. R. The Concept of Accessibility: A Search for an Operational Form. Regional Studies, Vol. 5, No. 2, 1971, pp. 101-107.

18. Morris, J. M., P. Dumble, and M. R. Wigan. Accessibility Indicators for Transport Planning. Transportation Research Part A, Vol. 13, No. 2, 1979, pp. 91-109.

19. Owen, A., and D. Levinson. Annual Accessibility Measure for the Twin Cities Metropolitan Area. MN/RC 2012-34. Minnesota Department of Transportation, Saint Paul, 2012.

20. Zhu, S. The Roads Taken: Theory and Evidence on Route Choice in the Wake of the I-35W Mississippi River Bridge Collapse and Reconstruction. $\mathrm{PhD}$ dissertation. University of Minnesota, Minneapolis, 2010.

21. Spear, B. D. Improving Employment Data for Transportation Planning. AASHTO, Washington, D.C., 2011.

22. Tilahun, N., and D. Levinson. Work and Home Location: Possible Role of Social Networks. Transportation Research Part A: Policy and Practice, Vol. 45, No. 4, 2011, pp. 323-331.

23. McFadden, D. The Measurement of Urban Travel Demand. Journal of Public Economics, Vol. 3, No. 4, 1974, pp. 303-328.

24. McFadden, D. Conditional Logit Analysis of Qualitative Choice Behavior. Academic Press, Waltham, Mass., 1974.

25. Menard, S. Coefficients of Determination for Multiple Logistic Regression Analysis. American Statistician, Vol. 54, No. 1, 2000, pp. 17-24.

26. Burnham, K. P., and D. R. Anderson. Model Selection and Multimodel Inference: A Practical Information-Theoretic Approach, 2nd ed. Springer-Verlag, New York, 2002.

27. Patterson, T. Lexus Lanes or Corolla Lanes? Spatial Use and Equity Patterns on the I-394 MnPASS Lanes. MS thesis. University of Minnesota, Minneapolis, 2008.

The Traveler Behavior and Values Committee peer-reviewed this paper. 